Neural, Parallel, and Scientific Computations 28 (2020) 83-93

\title{
DATA STREAMING AND SHARING BASED ON AUTHORIZED MULTIMEDIA USING ARTIFICIAL INTELLIGENCE MULTIMEDIA WIRELESS SENSOR NETWORKS
}

\author{
RAMACHANDRAN VARATHARAJAN \\ Bharath University, Chennai, India \\ Email : varathu21@gmail.com
}

\begin{abstract}
The rapid development of multimedia wireless sensor networks (MWSNs) and communication devices are becoming more and more common nowadays. Design and development of an effective security framework based on artificial intelligence (AI) for diversified wireless sensor networks and data transfer. The interpretation of the standard mechanism integrates that multimedia application network format and smart sensor data reliable data transmission. Blind Feature Learning (BFL) and Lightweight Physical Layer Verification (LPLA) Traditional security solutions and previous methods are not sufficient to effectively compute and address emerging security challenges. Besides, traditional top authentication ignores body layer protection, leading to serious privacy data leakage. The multimedia concept was discussed with the help of data sharing and data protection mechanisms and integrated framework of network multimedia simulation. Data authentication program combined with AI based-multimedia wireless sensor networks provides a distortion-free energy saving and real-time data flow. The project is compared to an unnoticed resource environment. Authorized Multimedia Streaming Data sharing (AMDSS) that recognizes the privacy of wireless multimedia sensors solution for data sharing and streamlining that can be realized. Performance test results show that intelligent verification program manual implementation improves the overall security of the multimedia network. Designed to protect both original data and data semantic information in the detection and method data sharing process.
\end{abstract}

Keywords: AI-Multimedia data sharing, privacy data, data leakage, streaming, data protection, Authorized data sharing, the security of the multimedia network.

\section{Introduction}

Wireless Multimedia Sensor Networks (WMSNs) produce immense sight and sound mass data. Due to the colossal size, the intuitive multimedia sensor node (MSN) can't store the sight and sound data made for a long time. For this condition, the flexible getting data game-plan can be used. Regardless, distant frameworks are vulnerable, where both MSN and versatile authority affirmation requires a practical security plan. At this moment, with the bewildering and good watching condition, essential data procured through standard distant sensor frameworks can't meet the overall necessities of regular checking. The direst task is to introduce biological watching practices reliant on the far off sensor to mastermind pictures, sound, video, and other intelligent media information data.

The Wireless Multimedia Sensor Network (WMSN) has appeared. WMSN is such a sensor sort out that fuses a camera, speaker sensor, and other environmental data variety limits. The Multimedia Wireless Sensor Networks (MWSNs), interface with sight and sound

Received March 1, 2020

www.dynamicpublishers.org
1061-5369 \$15.00 @ Dynamic Publishers, Inc. https://doi.org/10.46719/NPSC20202822 
sensor center points, including flexible modules based WMSN. Convenient distant sight and sound sensor frameworks are used in various checking applications, for instance, cutting edge condition, watchful traffic, splendid home, circumstance watching, and clinical prosperity watching. Convenient distant sight and sound sensor frameworks are used in various checking applications, for instance, cutting edge condition, watchful traffic, splendid home, circumstance watching, and clinical prosperity watching. Gathering works out, for instance, photos, accounts, sound data, and other sensor data social affairs, parties, sports competitions immense degree on various events data, are delivered on various relational associations. There are individuals on a comparable event who need to set up social associations and offer data about this event.

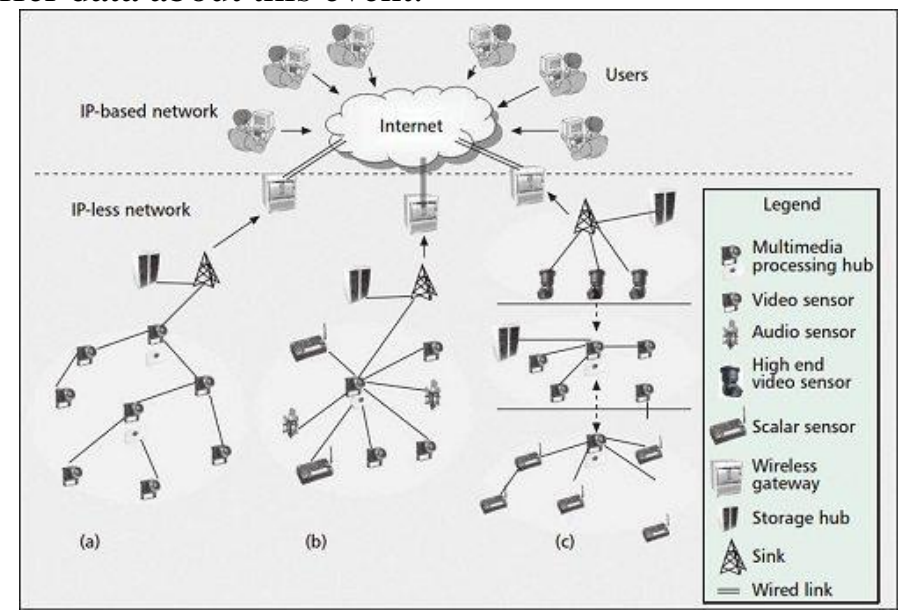

Figure 1: Wireless Multimedia Sensor Networks

[https://www.electronicsforu.com/technology-trends/introduction-wireless-multimediasensor-networks]

Figure 1 depicts the, In a distant sight and sound sensor sort out work, shared video reports, sound records and picture archives are sent over the recipient wire identified with an IP-based framework to pass on to customers by methods for the Internet. It is used as an inside point for limit purposes.

The Largest improvement of far off contraptions and far off developments has developed Multimedia Wireless Sensor Networks (MWSNs) to create a greater duty. An ordinary MWSN involves a huge number of pretty much nothing, low-charge sensor centers while the data is passed on to the target locale to accumulate interest. As a rule, MWSN is used in applications related to the perception and event of the board. Due to the everchanging substance of individuals and the various advancements of social activities, it is difficult to make sense of which part of all of the basic data activities to supervise different exercises, customer get-togethers. Blended media sensors can follow applications to get the most exact and positive information. In any case, the headway of gainful and advantageous responses for managing data correspondence through Wireless Multimedia Sensor Networks (WMSNs) is noteworthy taking into account the huge volume of data made by blendedmedia sensors. Assessment of the far off media sensor can simply part with from of framework limit, focus, and execution. This presents a combination of employments subject to far off intelligent media to compose sensors and their plan.

\section{Related Work}

Social applications, customers inviting associates, and sharing data, advancing individuals, and various activities need to execute any manual activities because of upsetting entirely unexpected data content. Social applications, customers inviting mates, and sharing data, developing individuals, and various activities need to execute any manual activities on 
account of upsetting very surprising data content. Social applications, customers inviting mates, and sharing data, developing individuals, and various activities need to execute any manual activities on account of upsetting very surprising data content. It is said that due to the proximity of media data it is essential to join complex weight counts. Wireless Multimedia Sensor Networks (WMSNs) Quality of Service (QOS) requires the framework to give steady quality. Center point to-end delay is a critical sign that WMSNs guarantee QoS. They have WMSNs where transmission bungles occur on the distant channel, making it difficult to acknowledge strong data move close by low center to-end delay. Sensor Networks Multimedia sensor frameworks make resource controls hard to see, and standard techniques can't adjust to the effects of blended media data transmission. Regardless, its universality is building up an immediate aftereffect of its inborn ordinary resident and military applications. Wireless Multimedia Sensor Networks (WMSNs) produce tremendous intelligent media mass data. Due to the immense size, the sight and multimedia sensor node (MSN) can't store the intelligent media data created for a long time. For this circumstance, flexible getting data combinations can be used.

Conveyed over Wireless Multimedia Sensor Networks (WMSNs) is a geographical administration program that stretches out system life cycle inclusion to guarantee an occasion, and keeps up availability between camera sensor (CS) hubs. Notwithstanding, closing down WMSNs in an automated situation makes hubs helpless against security assaults. The Wireless Vision Sensor Network (WVSN) can be used for following all bits of a city without the cost of running connections on the head of it. In any case, power usage should be the best way to deal with amass all the information accumulated by sensors and cameras that decline the ordinary holding up time. For a safe MWSN, the transmission and taking care of stream data ought to be discussed all around with a responsive standpoint. Due to standard data confirmation plans, figuring limits on sensor centers reliant on battery limits are available with information transmission and limit isn't fitting for MWSNs. A common far off sensor organizes game plan is an adaptable and secure data collection reliant on pressed acknowledgment. It is utilizing open key encryption advances to handle key courses and lessening the cost of contact identifying data combination in a particular region. Making far off sensor frameworks and resource controlled properties in an uninhabited space Sensor centers can do passing open sensors by methods for sensor centers to ensure that this information is then only accessible for a check. Multimedia Wireless Sensor Networks (MWSN) Low-Density Fertility Test Generator Team as a vertical vector tremendous resemblance occurs between the pictures got from the gathering cluster head $(\mathrm{CH})$. To take care of this issue, we have to decrease the bit mistake rate. The amount of terminals in a Multimedia wireless sensor network (MWSN) is one of the major limits when creating network code (NC) based counts with LDPC (low-density parity-check) codes, which directly impacts the size of the generator LDPC code and its decay show.

Gotten together with the versatile far off sight and sound sensor composes picture pressure work, the current methods don't think about the strong changes in getting ready execution and the territory of the joint exertion terminal. When managing picture pressure tasks, these methods often cause an obstruction and lead to data redistribution of those endeavors. Fitting fundamental specific structures and necessities is general, supporting different separated access to achieve the benefit of blend and action of info systems in different application circumstances. It offers an appropriate response for passing on home framework blended media content. In any case, standard far off frameworks may not benefit intelligent media data transmission over generally resource assignment millimeter-wave frameworks. To construct the yield of plant advancement, it is imperative to give impelled data on diseases of plants taught on the reason as for proposition got by farmers who follow up on it. As a result of the WMSN confinements, it is critical to design a direct system that 
can give high exactness and less multifaceted nature. Some related features of Wireless Multimedia Sensor Networks (WMSNs) rely upon the Advanced Real-Time Protocol (SPEED-RR) and QoS Guarantee Guide to ensure the idea of organization consistent execution reliant on the speed show used prevalently to tradeoff among continuous and power usage. A multi-target approach that gets ready for distant sight and sound sensor frameworks. Extending the components of an adaptable course will improve show redirection capacities in distant intelligent media sensor frameworks. Sight and sound data need to devour enough space for it. With some first-class sight and sound information, it is reasonably low regardless, when it shows up at the GB level and the intuitive media data organizes move speed. Wireless Sensor Networks, Technologies, and Applications a combination of distant sight and wireless multimedia sensor networks (WMSN) applications. Progress has been made in research regions where different structures and shows have been completed in WMSN.

\section{Materials and method}

Wireless Multimedia Sensor Networks (WMSNs) produce tremendous media mass data. Because of its tremendous size, the Multimedia Sensor Networks (MSN) can store sight and sound data that has been made for a long time. For this circumstance, versatile getting data combinations can be used. There is a prerequisite for an effective security plan for both MSN and adaptable get-together approval paid over the far off framework. Man-caused mental ability advancement can be used to improve execution and steadfast quality in these applications. Man-made intellectual competence computations Authorized Multimedia Streaming Data sharing (AMDSS) can be used to update sort out the heads, security, or data move coordinating in approved blended-media streams, provoking more strong frameworks used MWSN. It similarly discussed the opportunity of AMDSS using this AI to comprehend MWSN troubles and overhaul distant sensor frameworks and consideration, unfaltering quality. The properties, accessibility thickness, and execution were researched. A figuring for improving sight and sound participates in distant sensor frameworks, for instance, data sharing and acknowledgment shows. The base station is seen as arranged outside the framework district. MWSNs are gotten on account of delivering and smoothing out properties. When brought into the authentic condition, the ID accuracy of the impedance is inside and out decreased. To structure as a fitting flexible authorizer from a reasonable viewpoint paying little regard to the best situation. AMDSS figuring, to make it sensible for different assessments and correspondence conditions it is unsurprising with the approval model.

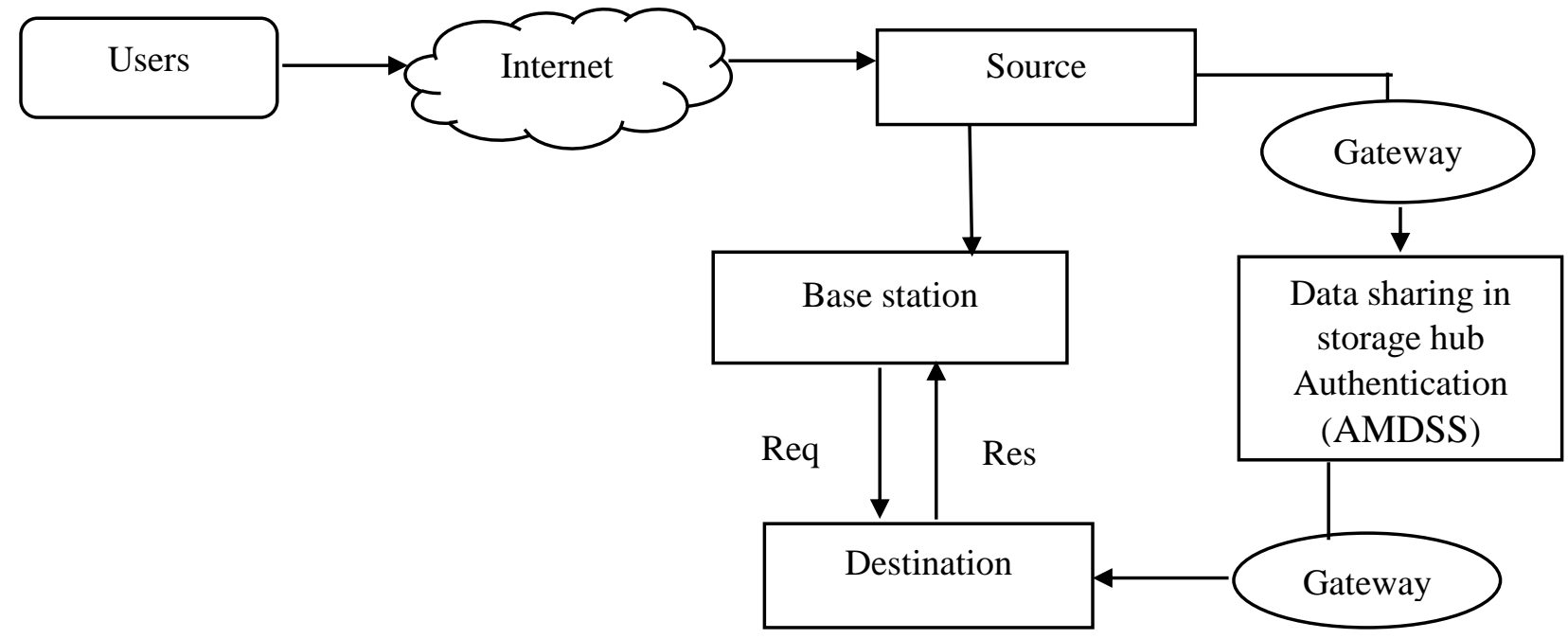

Figure 2: Block diagram 
Figure 2 depicts the movement of the source and objective data sharing between the Internets, in the limit community can be approving the steaming area, in the gateway watching out for the source and objective requesting and response.

\subsection{Cluster Streaming content delivery Multimedia}

Sight and sound Conference Services goes with coming about video benefits that are sensible for applications, which require incredibly low lethargy and can change the rundown (rate variety). Electronic component Class Multimedia is the most sensible for media applications where variable-rate streaming yield should, subsequently, can lessen its transmission rates, for instance, video constant and pocket adversity authentically on the sound and sight. These streams can, thusly, be assigned rate-flexible. Since two sorts of video conferencing gear are directly used in IP frameworks, different kinds of organization are required. Consistent shrewd organization devices are moreover used to make an electronic versatile video; Multimedia meeting organizations are contraptions that can move ongoing adaptable video.

\section{Algorithm: CSCDM}

Begin

Input: $\{\mathrm{SR}, \mathrm{CD}, \mathrm{CHl}$ dept. Tslots $\}$

If $(\mathrm{SR}<\mathrm{CD}) \wedge \mathrm{T}$-slot true then

$\mathrm{I} \rightarrow \mathrm{C}:\{$ Dcapt, internal gathering correspondence begins.

Else

$\mathrm{I} \rightarrow \mathrm{CSCDM}\{\mathrm{CReq}$, spare channel divide request. I set a clock TW for an answer.

CSCDM checks for any available IT times

True

$\mathrm{CSCDM} \rightarrow \mathrm{I}:\{$ Cres $\}$.

// I have a positive answer CSCDM sends.

Else

I keeping it together for its T opening.

End

If (tCRes TW) by then. Viably given out.

CSCDM \{ Dcapt, cover bunch correspondence starts.

Else

Get his T-space for checks

True

$\mathrm{I} \rightarrow \mathrm{ClH}:\{$ Dcapt $\}$.

End

End

End

Where, CSCDM-Cluster streaming substance movement Multimedia, CH-bunch spaces Transmit openings, sr-streaming resource, collection content transport. All gathering heads have CD spaces that consolidate both CDEPT, CLH, and CSCDM estimation. It is noteworthy here that a gathering head suggests the CLH of all part centers in a particular pack. In any case, a comparative gathering head is a CSCDM of another CL part terminal, and we find out that part center points to instate a CSCDM interest for channel allocation. Dept is resolved on the part center points that cross their Dcapt in solitude CLH. The requesting is reasonable.

\subsection{Authenticating Secure Link procedure}

Limit affiliation association or endorsement process reliant on the solidifying methodology. To meet different necessities for the organization's shortcomings with such an 
obstruction, the organization referencing endorsement is performed after the security standard is recognized. Along these lines, affirmation can't be impelled when making an association in correspondence employing motions. The organization affiliation is asserted when the sales are submitted.

\section{Algorithm}

Begin

- $\alpha, \leftarrow[\mathrm{m}, \lambda \mathrm{n}]$

$\cdot \mathrm{R} \leftarrow[\mathrm{m}, \lambda \mathrm{n}]$

If $\mathrm{rm}==\mathrm{rm}$ then

$(\alpha \mathrm{n}, \lambda \mathrm{n}, \mathrm{rm}) \rightarrow \mathrm{Rm}$

$$
\begin{aligned}
\text { If }[\alpha \mathrm{n}, \lambda \mathrm{m}]= & =\mathrm{A}[\alpha \mathrm{n}][\lambda \mathrm{n}] \text { then } \\
\alpha \mathrm{n} \longleftrightarrow & \longrightarrow \mathrm{rm} . \text { The gathering made among } \alpha \text { and } \mathrm{rm} \\
& \mathrm{Rm} \rightarrow \alpha \mathrm{n}:\{\mathrm{sr}, \text { slot created }\}
\end{aligned}
$$

Else

End if

$\alpha \mathrm{n}$ is unapproved

Else

End if

The requesting is for a substitute $r$ and discarded

$\alpha \mathrm{r} \rightarrow \mathrm{rm}:\{\mathrm{Cr}, \alpha \mathrm{r}$ respond with a test $\}$

If $\eta \mathrm{r}$ exists then

$\alpha \mathrm{n}$ is approved

Access granted

$$
\mathrm{Rm} \rightarrow \alpha \mathrm{n}:\{\mathrm{Cr}, \mathrm{rm} \text { responds with another test }\}
$$

Else

Access denied

an is unauthenticated

End if

If $\eta n$ exists then

$\mathrm{Rm}$ is authenticated

Data can be exchanged now

Else

End if

$\mathrm{Rm}$ is unauthenticated

\subsection{Authorized Multimedia Streaming Data sharing}

Our confirmation express instrument can be totaled into counts. Decreases sound difference in text or various pictures, estimation incitation transmission, and secure trade time. To deal with these issues, a channel assignment structure is a between-bunch development system. Because of a flood, the part center in a bundle has an available direct for post-appropriation if it transforms into a touching $\mathrm{CH}$. Exploratory results show that AMSDS gives a good level of security enhancement for the base framework.

\section{Algorithm-AMSDS}

Initialization:

A base station (BS) gives out tr to each moving toward $r$.

BS stores ID of each $r$ in a table.

BS allots $\lambda i$ to each $r$. 


\author{
Confirmation: Base Station-Cluster Head \\ For $\mathrm{r}=1$ : do \\ For $\mathrm{s}=1$ do
}

Input a[r] [s]. IDNB and $\lambda \mathrm{i}$ are stored in $\mathrm{a}[\mathrm{r}][\mathrm{s}]$

$\mathrm{R} \rightarrow \mathrm{BS}:\{$ ctr $\mathrm{r}$ : control bundles convey by each $\mathrm{r}\}$

BS scrambles AMSDS with $\tau$ chi to makers ID.

In case AMSDS matches, by then. A match is found

$\mathrm{CHi}$ recuperate AMSDS and $\mathrm{A}[\mathrm{r}][\mathrm{s}]$.

End if

$\mathrm{CHi} \rightarrow$ BS: $\{$ ACK: control pack impart by each $\mathrm{CHi}\}$

BS checks for IDBS in ACK.

CHi authenticated.

If IDBS matches, by then. A match is found

\title{
End if
}

End for

End for

Approval: Within the Cluster

$\mathrm{CHi} \rightarrow \mathrm{r}:\{$ control package containing AMSDS $\}$

$\mathrm{R}$ retrieves AMSDS.

CHi retrieves ID and AMSDS.

If ID $==\mathrm{A}[\mathrm{R}][0]$ and AMSDS matches then

check the validity of $r$

$\mathrm{CHi} \rightarrow \mathrm{r}:\{\gamma$ challenge: the mixed trial of $\mathrm{CHi}\} \cdot \gamma$ challenge is used to

Else

End if

$\mathrm{R}$ is unapproved and s Req $\mathrm{r}$ discarded.

If both matches then

Formation.

$\mathrm{R}$ Becomes a section center point of $\mathrm{CH} \mathrm{r}$ Authentic Cluster

Else

$$
\mathrm{CHi} \rightarrow \mathrm{r}:\{\gamma \text { response: encoded response of } \mathrm{CH} \mathrm{r}\}
$$

CHi conveys $\gamma$ response containing $\eta \mathrm{i}$

End if

$\mathrm{R}$ is unapproved and restricted from correspondence to shape a pack.

If both matches then

$\mathrm{R}$ transforms into a section center of $\mathrm{CHi}$

$\mathrm{CHi}$ allocates $\mathrm{T}$ - slots to $\mathrm{r}$

Else

End if

$\mathrm{CHi}$ is unauthorized

Where, AMSDS-Authorized Multimedia Streaming data sharing, CHI-Cluster Head, $\mathrm{R}, \mathrm{s}$-variable-spaces Transmit Slots, IDBS-Identity base station, If the $\eta \mathrm{R}$ bond is made, it infers that the proper reaction is gotten from the legal structure. Presently, $R$ agrees that $R$ and $\mathrm{C}$ are the keys to shared affirmation and the customary gathering key $\mathrm{S}$ for data move. Each time that the method of shared affirmation happens, I need to incorporate a potential $\mathrm{CHI}$. At the point when affirmed, each I center transforms into its CHI part provoking the creation of a sheltered pack. Presently, the inertia stage begins and each $r$ moves its got data to its CHI Authenticated. 


\section{Result and discussion}

The test was played out a couple of times at different space densities and various levels to ensure better discretion and genuine gathering. Stacked with scalar indicating the most extraordinary for consecutive models from every data channel that can be appreciated with the time slot. This connection relies upon the total number of data sent and the number of pieces to send such information. It contemplates AMSDS against existing Blind Feature Learning (BFL) and Lightweight Physical Layer Verification (LPLA) execution estimation of rules, for instance, contact revelation execution, vital time, and yield and pocket move rate.

Table 1: Simulation Parameters

\begin{tabular}{|c|c|}
\hline Parameters & Value \\
\hline Simulation Tool & Dot net \\
\hline Network security & Multimedia WSN \\
\hline Transferring data & 250 \\
\hline Data size & $100 \mathrm{mb}$ \\
\hline Verification method & Authentication Data sharing Method \\
\hline
\end{tabular}

Table 1 shows, the described characteristics and assessment limits of the proposed methodology. The capability of multi-item data was assessed, the check Data sharing Method in the Storage strategy.

\subsection{Analysis of Storage performance}

Limit contraptions, especially hard drives for limit operational activities. This standardizes the metric estimation by taking a gander at the drive being attempted by its show. Limit Performance Indicators It is perfect to evaluate the capability of their storing systems. The limit demonstrated is regularly evaluated the extent that the breaking point, yield, and usage.

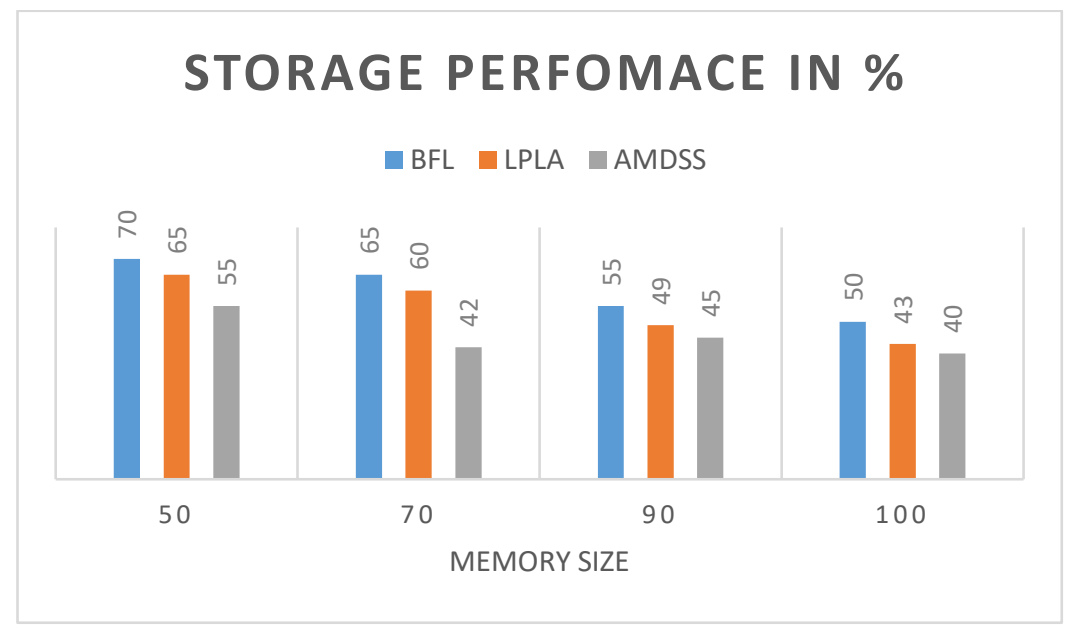

Figure 3: Analysis of storage performance

Figure 3 portrays the proportion of memory that is considered to diminish the accumulating execution assignment. The current BFL figuring has a limited capability of $55 \%$, and the LPLA has limited adequacy of $45 \%$. As proposed, the memory size reduction is $30 \%$. 


\subsection{Analysis of the Performance Accuracy}

Figures the extent, following the intuitive media data sharing desire for execution during the show assessment. Assessment with the teaching of various data sharing properties, similarly as finds related to the precision of data moves close and the exercises of thoughts. The impact of execution precision and data sharing exactness is high and utilization security is high.

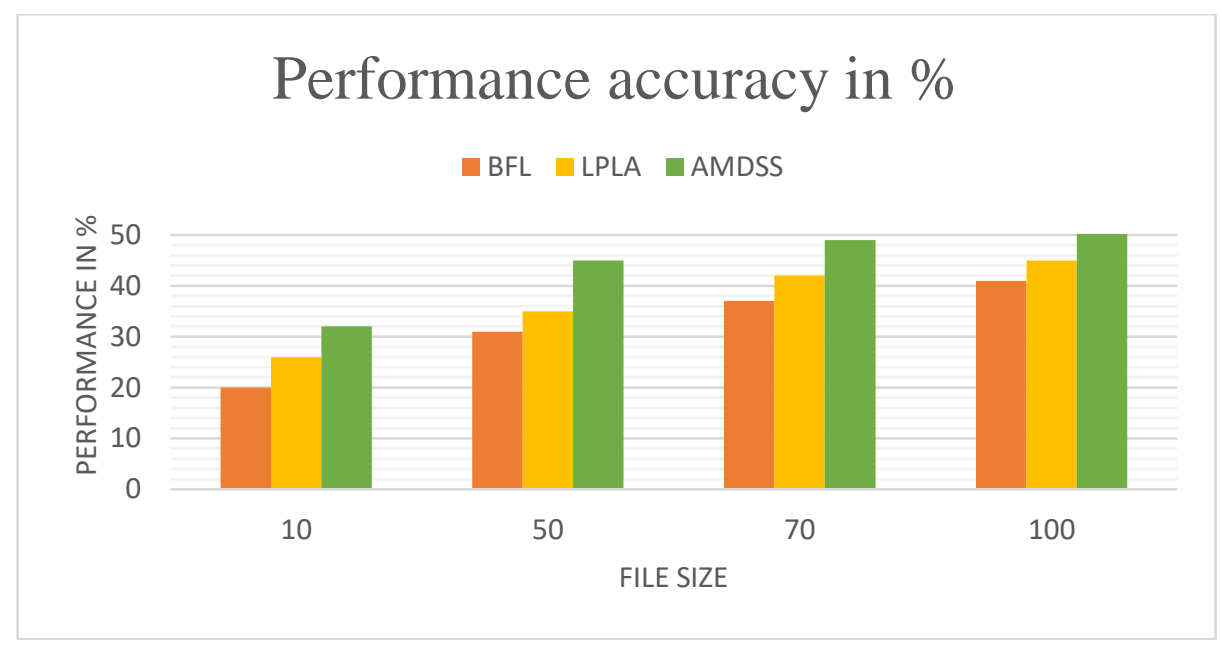

Figure 4: Analysis of performance accuracy

Figure 4 depicts the introduction accuracy for the current method for BFL $45 \%$ in LPLA $41 \%$ in the show at the low-execution level.in the proposed methodology for AMDSS is $52 \%$ of high secure execution precision for the record size.

\subsection{Analysis of the Time complexity}

Complex time can be easily evaluated by running the endeavor time. You can see the most extraordinary number of rough advances that can make a stunning application. Custom organizations may have extended individual and spotlights on those that run increment, scattering, etc., unlimited activities, and the most specific proportion of time left. This limit is called quality. The amount of accepted organizations depends upon the specific data. Time commonly depends upon how one piece of the introduction data depends. This is constantly the proportion of data, yet it can moreover be the size of a square display or the estimation of some data factors.

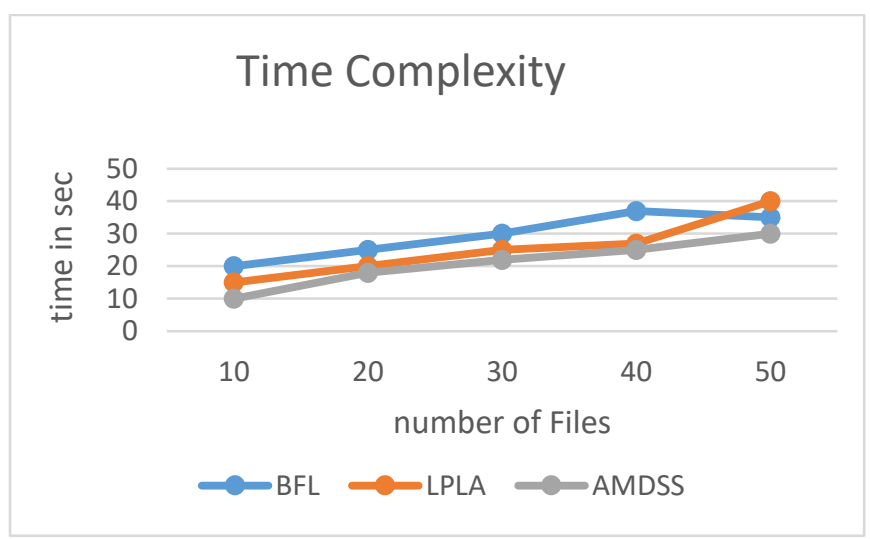

Figure 5: Time complexity 
Figure 4.3 depicts the defer execution is resolved reliant on the number of data records to be passed on to the objective inside a period. Taking a gander at the past time unconventionality decays the proposed method. In the time complexity of the number of records processes the $40 \mathrm{sec}$ for BFL ascertaining the course of occasions evaluates the base of 50 records in $40 \mathrm{sec}$ for LPLA, and AMDSS in $30 \mathrm{Sec}$ the proposed procedure diminishes the time capriciousness.

\section{Conclusion}

The far off intelligent media organize the game plan in like manner has feasible importance as the man-made thinking based security affirmation proposed in this document. The proposed procedure, made by an AMDSS shows the data sharing of a securely seen terminal and is used in a target intend to measure at media sort out core interests. The AMDSS program can update amassing, security execution, and reduce the association between the various properties of the WSNs. Data section structure and the fragment time model to make a specific event model conflict with the distant secure blended-media sort out.

\section{References}

1. Han, F., Zhang, L., Yu, X., Wang, G., \& Li, X.-Y. (2019). SHAD: Privacy-Friendly Shared Activity Detection And Data Sharing. 2019 IEEE 16th International Conference on Mobile Ad Hoc And Sensor Systems (MASS).

2. Yadav, V., \& Arora, G. (2019). Analysis of Wireless Multimedia Sensor Network. 2019 2nd International Conference on Power Energy, Environment, And Intelligent Control (PEEIC).

3. N. Abbas And F. Yu, "Performance Analysis Of End-To-End Delay and Reliability For Linear Wireless Multimedia Sensor Networks," 2017 IEEE 3rd Information Technology And Mechatronics Engineering Conference (ITOEC), Chongqing, 2017, Pp. 554-558

4. Deb, Moumita \& Choudhury, Abantika. (2018). A Review Of Wireless Multimedia Sensor Network And Existing Routing Protocols. International Journal Of Computer Sciences And Engineering. 6. 343-358. 10.26438/Ijcse/V6i3.343358.

5. Usman, M., Jan, M. A., He, X., \& Chen, J. (2018). A Mobile Multimedia Data Collection Scheme For Secured Wireless Multimedia Sensor Networks. IEEE Transactions On Network Science And Engineering, 1-1.

6. G. Mali And S. Misra, "Trust: Trust-Based Distributed Topology Management For Wireless Multimedia Sensor Networks," IEEE Transactions On Computers, Vol. 65, No. 6, Pp. 19781991, 2016.

7. J. P. J. Peixoto And D. G. Costa, "Wireless Visual Sensor Networks For Smart City Applications: A Relevance-Based Approach For Multiple Sinks Mobility," Future Generation Computer Systems, Vol. 76, Pp. 51-62, 2017

8. M. Usman, M. A. Jan, X. He, And P. Nanda, "Data Sharing In Secure Multimedia Wireless Sensor Networks,” In Trustcom/Bigdatase/I SPA, 2016 IEEE. IEEE, 2016, Pp. 590-597. 
9. P. Zhang, S. Wang, K. Guo, And J. Wang, "A Secure Data Collection Scheme Based On Compressive Sensing In Wireless Sensor Networks," Ad Hoc Networks, Vol. 70, Pp. 7384, 2018.

10. S. Kumari, M. K. Khan, And M. Atiquzzaman, "User Authentication Schemes For Wireless Sensor Networks: A Review,” Ad Hoc Networks, Vol. 27, Pp. 159-194, 2015.

11. Chateau, Y., Maslouhi, I., Ghoumid, K., \& Ar-Reyouchi El Miloud. (2018). Performance Enhancement Of Wireless Sensor Networks Using An Efficient Coding Approach. 2018 6th International Conference On Multimedia Computing And Systems (ICMCS).

12. M. Hammouti, E. M. Ar-Reyouchi, K. Ghoumid, And A. Lichioui, "Clustering Analysis Of Wireless Sensor Network Based On Network Coding With Low-Density Parity Check," International Journal Of Advanced Computer science And Applications (Ijacsa), Vol. 7, No. 3, P. 2016, 2016.

13. HE LI, QINGLEI QI, XINJIANG LIU, PAN ZHAO2, AND YANG YANG, "Mobile Wireless Multimedia Sensor Networksimage Compression Task Collaboration Based On Dynamic Alliance", IEEE Access-2020, Pp-86024 - 86037.

14. F. Kong, Y. Zhou, And G. Chen, "Multimedia Data Fusion Method Based Wireless Sensor Network In Intelligent Transportation System,' Multimedia tools Appl., Vol. 4, Pp. 1-13, Apr. 2019.

15. B. Ma, H. Shah-Mansouri, And V. Wong, "Multimedia Content Delivery In Millimeter Wave Home Networks,' IEEE Trans. Wireless Commun., Vol. 15, No. 7, Pp. 4826-4838, Jul. 2016.

16. K. Indumathi, R. Hemalatha, S. A. Nandhini, And S. Radha, “Intelligent Plant Disease Detection System Using Wireless Multimedia Sensor Networks,' In Proc. Int. Conf. Wireless Commun., Signal Process. Netw. (Wispnet), Mar. 2017, Pp. 1607-1611.

17. Z. Yu And B. Lu, “A Multipath Routing Protocol Using Congestion Control In Wireless Multimedia Sensor Networks,', Peer-To-Peer Netw. Appl., Vol. 12, No. 6, Pp. 1585-1593, Nov. 2019.

18. R. Borawake-Satao And R. Prasad, "Mobility Aware Multi-Objective Routing In Wireless Multimedia Sensor Network,' Multimedia Tools Appl., Vol. 78, No. 23, Pp. 32659-32677, Dec. 2019

19. N. Ma, "Distributed Video Coding Scheme Of Multimedia Data Compression Algorithm For Wireless Sensor Networks,' EURASIP J. Wireless Commun. Netw., Vol. 2019, No. 1, P. 254, Dec. 2019.

20. Doorwar, M., \& Malathi, P. (2018). Comparison Of Protocol Based On Architecture In Wireless Multimedia Sensor Network. 2018 IEEE Global Conference On Wireless Computing And Networking (GCWCN). 